Article

\title{
Effectiveness of Collagen Membrane in the Treatment of Schneiderian Membrane Perforation
}

\author{
Jae-Yeol Lee ${ }^{1,2}$, Jin-Ju Kwon ${ }^{1}$, George K. Sándor ${ }^{3,4}$ and Yong-Deok Kim 1,2,* \\ 1 Department of Oral and Maxillofacial Surgery, School of Dentistry, Pusan National University, \\ Yangsan 50612, Korea; omsljy@pusan.ac.kr (J.-Y.L.); jinju1004_kr@naver.com (J.-J.K.) \\ 2 Institute of Translational Dental Sciences \& Dental Research Institute, Pusan National University, \\ Yangsan 50612, Korea \\ 3 Department of Oral and Maxillofacial Surgery, Oulu University Hospital and Medical Research Center, \\ University of Oulu, 90014 Oulu, Finland; sandor_george@hotmail.com \\ 4 BioMediTech, Institute of Biosciences and Medical Technology, University of Tampere, \\ 33100 Tampere, Finland \\ * Correspondence: ydkimdds@pusan.ac.kr; Tel.: +82-55-360-5100
}

Received: 5 March 2019; Accepted: 10 April 2019; Published: 11 April 2019

check for updates

\begin{abstract}
Schneiderian membrane perforation (SMP) is the most common complication encountered during sinus lift procedures. SMPs should be managed to prevent loss of the valuable bone graft and bone substitute materials. A fast-resorbing collagen membrane (CM) is a soft, white, pliable, and nonfriable sponge used in dental surgery. The purpose of this study was to evaluate the clinical outcomes of SMP repair using CMs when an SMP had occurred during a sinus lifting procedure. The patients were divided into three groups according to the size of the SMPs during the sinus lift procedure. (A) Group 1: there was no perforation of the Schneiderian membrane but the membrane was weakened (or thinned) and repaired using CMs. (B) Group 2: the SMP was small to medium in size $(<10 \mathrm{~mm}$ ) and repaired using CMs and fibrin adhesive (FA). (C) Group 3: the SMP was large in size $(>10 \mathrm{~mm}$ ) and repaired using a collagen plug. The negative control group consisted of patients who did not have any SMP (Control) and these patients were randomly selected. Orthopantomographic X-rays taken before surgery, 2-3 days following surgery, and over 6 months after surgery (follow-up) were used to evaluate the acquired bone height according to groups. Bone heights were measured from the crestal bone at the planned implant placement sites. Clinical outcomes, including implant success and complications according to repair method, were also investigated. There was no significant difference in bone heights between the groups with the exception of group 3 . The overall implant survival rate was $100 \%$ for implants placed in sinuses with frank SMPs or weakened sinus membranes. The CM is applicable for small-to-moderate perforations or sinus membranes which have been weakened or thinned during sinus lift procedures.
\end{abstract}

Keywords: sinus lift procedure; Schneiderian membrane; perforation; collagen membrane

\section{Introduction}

With the increased popularity of dental implant therapy to replace missing teeth, the need has arisen for a reliable method to provide patients with bony support for these implants in cases where the alveolar ridge volume is insufficient for implant placement. In particular, bone remodeling following tooth extraction or trauma and maxillary sinus pneumatization often create a clinical challenge for dental implant placement in the posterior maxilla. First introduced by Tatum [1] and then published by Boyne and James [2], sinus lifting has become a predictable procedure in the rehabilitation of vertical bone deficiency in the posterior maxillary region [3]. 
Although the maxillary sinus lift procedure prior to dental implant placement is a predictable and safe procedure, complications such as Schneiderian membrane perforation (SMP), excessive bleeding, and infection have been reported during the procedure and may influence the outcome of treatment [4]. The most common complication is SMP. According to previous studies, the weighted average for the incidence rate of SMP was 23.5\%, ranging from 3.6\% to 41.8\% [5]. Fugazzotto and Vlassis [6] indicated that SMPs are not considered a reason to discontinue sinus lift procedures but should be addressed by properly isolating and repairing the SMP. Repair could include folding of the sinus membrane itself, covering the SMP with an absorbable membrane, or careful suturing. As an alternative, the use of fibrin adhesive (FA) for repair of perforations has been advocated [4,7].

Among these repair methods, the collagen membrane (CM) is the most widely used, but there is little research on the effectiveness of CMs in the management of SMPs during maxillary sinus lift procedures [8]. This study aimed to evaluate the clinical outcomes of SMP repair using a CM when such an SMP had occurred during a sinus lifting procedure.

\section{Materials and Methods}

\subsection{Patients}

This retrospective review included all patients who had received maxillary sinus augmentation procedures via a lateral window technique by the same surgeon from the period of March 2014 to October 2017 in Pusan National University Dental Hospital. The following patients were excluded: those with unstable systemic diseases, uncontrolled metabolic diseases, or a habit of smoking $\geq 10$ cigarettes/day; those who were receiving medication that altered bone healing, such as bisphosphonates; and those with a history of chemotherapy or radiative therapy performed in the oral and maxillofacial region. The patients were divided into the following groups according to their sinus mucosal status during the sinus lift procedures:

(A) Group 1: No SMP but the membrane was weakened (or thinned) during sinus lift procedure;

(B) Group 2: SMP was small to medium in size $(<10 \mathrm{~mm})$ during sinus lift procedure;

(C) Group 3: SMP was large in size $(>10 \mathrm{~mm})$ during sinus lift procedure;

(D) Control: Sinus membrane was not perforated during sinus lift procedure.

\subsection{Maxillary Sinus Lift Procedure}

All patients signed a written consent form before the surgery. After performing a crestal incision and a mucoperiosteal flap elevation, the lateral wall of the sinus was exposed. During the process of forming an oval bony window using a round bur, the sinus membrane was elevated.

Once SMPs were identified visually, repair was achieved with a fast-resorbing CM (Colla-tape ${ }^{\circledR}$, Zimmer, Palm Beach Gardens, FL, USA), a collagen plug (CP, Ateloplug ${ }^{\circledR}$, Bioland, Cheonan, Korea), or FA (Tisseel ${ }^{\circledR}$, Baxter, Deerfield, IL, USA) according to the perforation size. In small-to-medium perforations (<10 mm, Group 2), a fast-resorbing CM was placed dry into the sinus cavity and directly onto the SMP. After the application of FA on the CM, sinus augmentation was continued with placement of bovine bone materials (Bio-oss ${ }^{\circledR}$, Geistrich, Zürich, Swiss) that had been hydrated with sterile saline (Figure 1). In cases of large SMPs (> $10 \mathrm{~mm}$, Group 3), in order to avoid failure of the sinus membrane repairs or graft leakage into the maxillary sinus, the authors did not insert graft materials. Instead of graft materials, a CP was inserted into the sinus after making the perforation as small as possible without additional bone graft (Figure 2). Implant placements were performed if adequate initial stability could be obtained. When there was no SMP but a weakened or partially irritated membrane (Group 1), bone graft after application of CM was performed. After placement of the bone grafts, all lateral access windows were covered once again with their original bony windows. Primary wound closure was achieved in all cases. Simultaneous or delayed bone grafting, implant success, and complications according to repair method were investigated. 


\subsection{Radiographic Examination}

Orthopantomographic X-rays taken before surgery (pre op), 2-3 days after surgery (post op), and between 6 to 12 months after surgery (follow-up) were used to evaluate the acquired bone height according to groups. Bone heights from the crestal bone at the planned implant placement sites were measured and evaluated (Figures 3 and 4). For a negative control group, those patients who did not have SMPs (Control) were selected if there was sufficient information, including radiographs at appropriate time points for this study, and their bone heights were measurable.
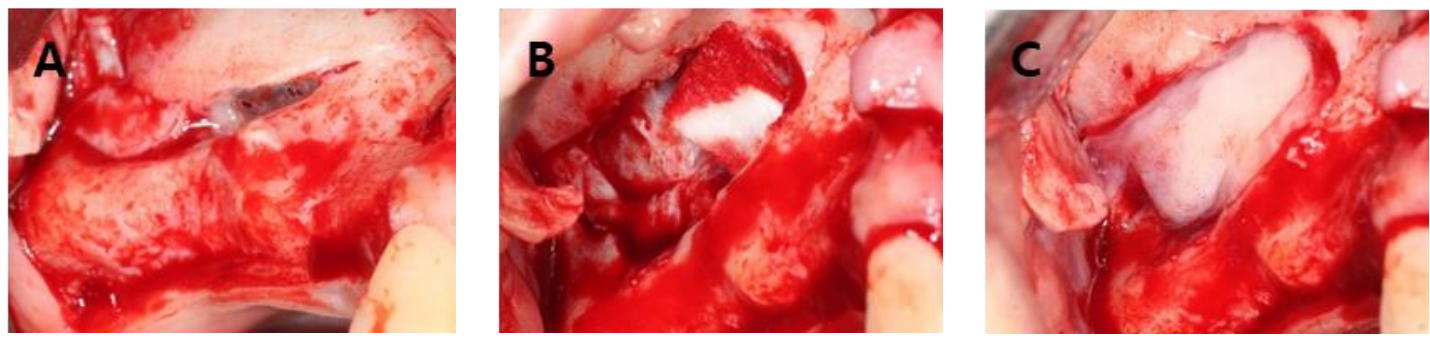

Figure 1. Small perforation of sinus membrane during sinus floor elevation procedure. (A) Small perforation during surgical window. (B) Repair with collagen membrane (CM). (C) Bone graft and repositioning of bony window.
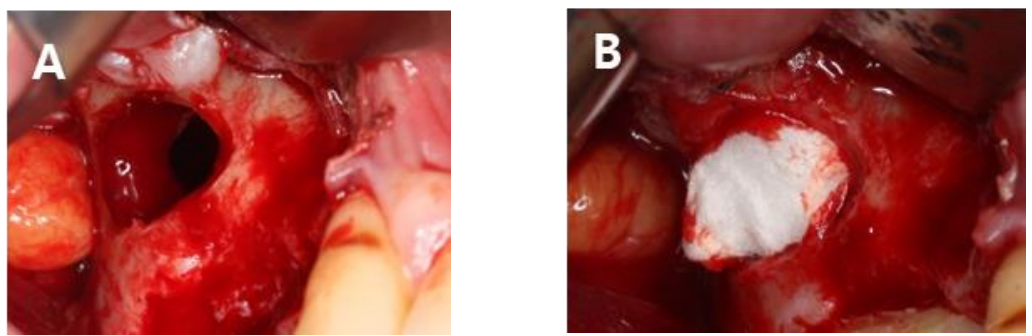

Figure 2. Large perforation of sinus membrane during sinus floor elevation procedure. (A) Large perforation of membrane ( $>10 \mathrm{~mm}$ ). (B) Insertion of collagen plug $(\mathrm{CP})$.
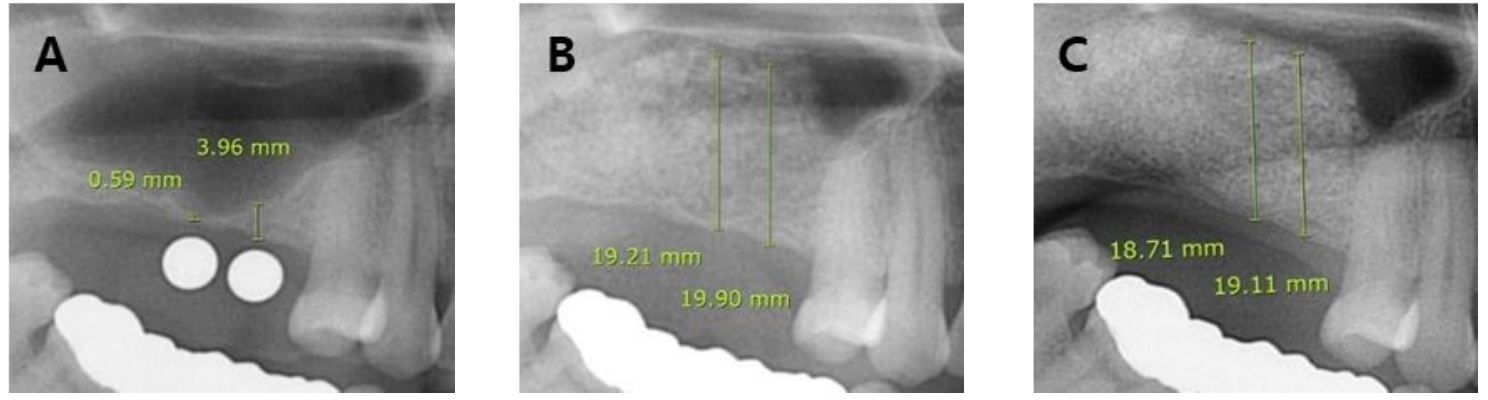

Figure 3. Bone height from the crestal bone at the planned implant placement site. (A) Pre op. (B) Post op. (C) Follow-up. 

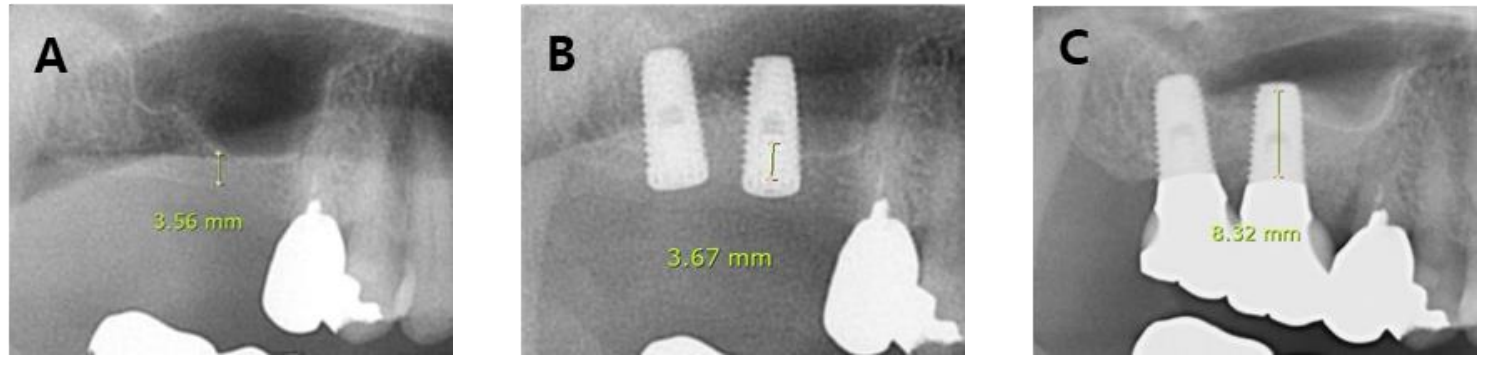

Figure 4. Bone height measured from the crestal bone of group 3. (A) Pre op. (B) Post op. (C) Follow-up.

\subsection{Statistical Analysis}

Data were analyzed using statistical software (IBM Corp. SPSS Statistics Version 22.0, Chicago, IL, USA, 2013). The normality test used the Kolmogorov-Smirnov test. Data are presented as means \pm standard deviations. Significance was determined using two-way repeated-measures ANOVA. Significance for pairwise comparison was determined with the Bonferroni post hoc test.

\section{Results}

One hundred and ninety cases were identified, resulting in a total of 250 lateral window sinus augmentations, of which 23 SMPs $(9.2 \%)$ were noted. There were 15 cases $(6 \%)$ where there was no perforation of the sinus membranes but they were weakened. Among patients with perforations, 11 sinuses (47.8\%) had small-to-medium SMPs, and 12 sinuses (52.2\%) had large SMPs. Demographic characteristics of each group are described in Table 1. All patients with small-to-medium perforations received simultaneous bone grafting, and 43 implants were inserted. Only one complication (periimplantitis) was observed. In patients with large perforations, one patient had received an additional sinus lift on the same operative site, while other patients were able to receive implant placement without additional bone grafting. All patients with sinus membranes that were not perforated but weakened were treated with simultaneous bone grafting after reinforcement with a CM. A total of 83 dental implants were placed in augmented sinuses with perforated or weakened sinus membranes. The overall implant survival rate was 100\% for implants placed in perforated or weakened sinuses, and four periimplantitis cases $(4.8 \%)$ were observed during follow-up periods (Table 2).

Table 1. Demographic characteristics of patients.

\begin{tabular}{|c|c|c|c|c|c|c|c|c|c|}
\hline \multirow[t]{2}{*}{ Characters } & \multirow[t]{2}{*}{.. } & \multicolumn{2}{|c|}{ Group 1} & \multicolumn{2}{|c|}{ Group 2} & \multicolumn{2}{|c|}{ Group 3} & \multicolumn{2}{|c|}{ Control } \\
\hline & & n (\%) & $\begin{array}{l}\text { Average } \\
\pm \text { SD }\end{array}$ & n (\%) & $\begin{array}{c}\text { Average } \\
\pm \text { SD }\end{array}$ & n (\%) & $\begin{array}{l}\text { Average } \\
\pm \text { SD }\end{array}$ & n (\%) & $\begin{array}{c}\text { Average } \\
\pm \text { SD }\end{array}$ \\
\hline \multirow{2}{*}{ Gender } & $\mathrm{F}$ & $11(73.3)$ & & $4(36.4)$ & & $4(33.3)$ & & 18 (48.6) & \\
\hline & M & $4(26.7)$ & & $7(63.6)$ & & $8(66.7)$ & & $19(51.4)$ & \\
\hline \multirow{2}{*}{ Age } & $<65$ & 13 (86.7) & $55.8 \pm$ & $9(81.8)$ & $51.181 \pm$ & $8(66.7)$ & $57.916 \pm$ & $29(78.4)$ & $57.378 \pm$ \\
\hline & $\geq 65$ & $2(13.3)$ & 12.946 & $2(18.2)$ & 9.042 & $4(33.3)$ & 14.368 & $8(21.6)$ & 10.034 \\
\hline \multirow{2}{*}{ Sinus Septum } & Yes & $3(20)$ & & $6(54.5)$ & & $5(41.7)$ & & 7 (18.9) & \\
\hline & No & $12(80)$ & & $5(45.5)$ & & $7(58.3)$ & & 30 (81.1) & \\
\hline \multirow{2}{*}{$\begin{array}{l}\text { Residual Bone } \\
\text { Height }\end{array}$} & $<4.0$ & $13(86.7)$ & $2.037 \pm$ & $10(90.9)$ & $2.036 \pm$ & $10(83.3)$ & $2.200 \pm$ & 29 (78.4) & $2.270 \pm$ \\
\hline & $\geq 4.0$ & $2(13.3)$ & 1.436 & $1(9.1)$ & 1.415 & $2(16.7)$ & 1.351 & $6(22.2)$ & 1.441 \\
\hline \multirow[t]{2}{*}{ Alveolar Width } & $\begin{array}{l}1-2 \text { teeth } \\
\text { gap }\end{array}$ & $8(53.3)$ & \multirow{2}{*}{$\begin{array}{l}2.6 \pm \\
0.910\end{array}$} & $4(36.4)$ & $\begin{array}{c}3.000 \pm \\
0.894\end{array}$ & $5(41.7)$ & $\begin{array}{l}2.916 \pm \\
1.083\end{array}$ & 30 (81.1) & $\begin{array}{c}2.000 \pm \\
0.707\end{array}$ \\
\hline & $\begin{array}{l}\text { 3-4 teeth } \\
\text { gap }\end{array}$ & $7(46.7)$ & & $7(63.6)$ & & $7(58.3)$ & & $7(18.9)$ & \\
\hline
\end{tabular}


Table 2. Summary regarding progress of repair according to perforation size. (All complications were periimplantitis during follow-up period. Abbreviations; CM: Collagen membrane, FA: Fibrin adhesive, CP: Collagen plug, G: Group, BS: Bone substitute).

\begin{tabular}{|c|c|c|c|c|c|c|c|c|}
\hline G & Perforation & Repair & $\mathbf{N}$ (site) & Grafting of BS (site) & \multicolumn{3}{|c|}{ Implant (Patient/implant) } & Complication \\
\hline \multirow{3}{*}{1} & \multirow{3}{*}{ Weakened } & \multirow{3}{*}{$\mathrm{CM}$} & \multirow{3}{*}{15} & \multirow{3}{*}{ simultaneous } & \multirow{3}{*}{15} & Simultaneous & $14 / 34$ & 1 \\
\hline & & & & & & Delayed & $1 / 2$ & 0 \\
\hline & & & & & & None & & 0 \\
\hline \multirow{3}{*}{2} & \multirow{3}{*}{$\begin{array}{c}\text { Small } \\
(<10 \mathrm{~mm})\end{array}$} & \multirow{3}{*}{$\mathrm{CM}+\mathrm{FA}$} & \multirow{3}{*}{11} & \multirow{3}{*}{ simultaneous } & \multirow{3}{*}{11} & Simultaneous & $1 / 2$ & 0 \\
\hline & & & & & & Delayed & $8 / 23$ & 1 \\
\hline & & & & & & None & 2 & 0 \\
\hline \multirow{7}{*}{3} & \multirow{7}{*}{$\begin{array}{c}\text { Large } \\
(>10 \mathrm{~mm})\end{array}$} & \multirow{7}{*}{$\mathrm{CP}$} & \multirow{7}{*}{12} & \multirow{5}{*}{ delayed } & \multirow{4}{*}{1} & \multirow{2}{*}{ Simultaneous } & 0 & 0 \\
\hline & & & & & & & 0 & 0 \\
\hline & & & & & & Delayed & 0 & 0 \\
\hline & & & & & & None & 1 & 0 \\
\hline & & & & & \multirow{3}{*}{11} & Simultaneous & $6 / 13$ & 1 \\
\hline & & & & \multirow[t]{2}{*}{ none } & & Delayed & $4 / 9$ & 1 \\
\hline & & & & & & None & $1 / 0$ & 0 \\
\hline
\end{tabular}

On orthopantomographic images, the preoperative mean height of residual bone varied from 3.88 to $5.53 \mathrm{~mm}$ in all groups, but there was no significant difference between groups. In post op and follow-up time, there was a statistically significant difference between group 3 and all other groups. The bone height of group 2 was significantly lower than the control group in post op time. There was no significant difference between the groups except for group 3 in follow-up time (Tables 3 and 4). See Figure 5 and visualize Tables 3 and 4 .

Table 3. Bone height from crestal bone at the planned implant placement site (mm).

\begin{tabular}{ccccc}
\hline Group & Pre op & Post op & Follow-up & Source: $\mathbf{F}(\mathbf{p})$ \\
\hline 1 & $4.08 \pm 2.55$ & $14.94 \pm 3.00$ & $13.00 \pm 2.40$ & Group: $52.97(<.001)$ \\
2 & $4.92 \pm 2.55$ & $13.81 \pm 3.61$ & $12.65 \pm 3.43$ & Time: $203.67(<.001)$ \\
3 & $3.88 \pm 2.21$ & $3.82 \pm 2.19$ & $4.99 \pm 2.59$ & Group*Time: $11.67(<.001)$ \\
Control & $5.53 \pm 3.29$ & $16.00 \pm 3.63$ & $13.22 \pm 4.09$ & \\
\hline
\end{tabular}

Table 4. Mean difference between groups according to measurement time. (*: Bonferroni post hoc test $(p=0.05 / 3)$.)

\begin{tabular}{cccc}
\hline Group & Pre op & Post op & Follow-Up \\
\cline { 2 - 4 } & \multicolumn{3}{c}{ Diff. (M \pm SD) } \\
\hline Group 1 - Group 2 & $-0.84 \pm 0.69$ & $1.12 \pm 0.89$ & $0.34 \pm 0.78$ \\
Group 1 - Group 3 & $0.19 \pm 0.72$ & $11.11 \pm 0.81^{*}$ & $8.00 \pm 0.73^{*}$ \\
Group 1 - Control & $-1.45 \pm 0.67$ & $-1.05 \pm 0.75$ & $-0.22 \pm 0.65$ \\
Group 2 - Group 3 & $1.04 \pm 0.74$ & $9.99 \pm 0.96^{*}$ & $7.66 \pm 0.96^{*}$ \\
Group 2 - Control & $-0.61 \pm 0.72$ & $-2.18 \pm 0.84^{*}$ & $-0.56 \pm 0.91$ \\
Group 3 - Control & $-1.65 \pm 0.82$ & $-12.72 \pm 0.67^{*}$ & $-8.23 \pm 0.78^{*}$ \\
\hline
\end{tabular}




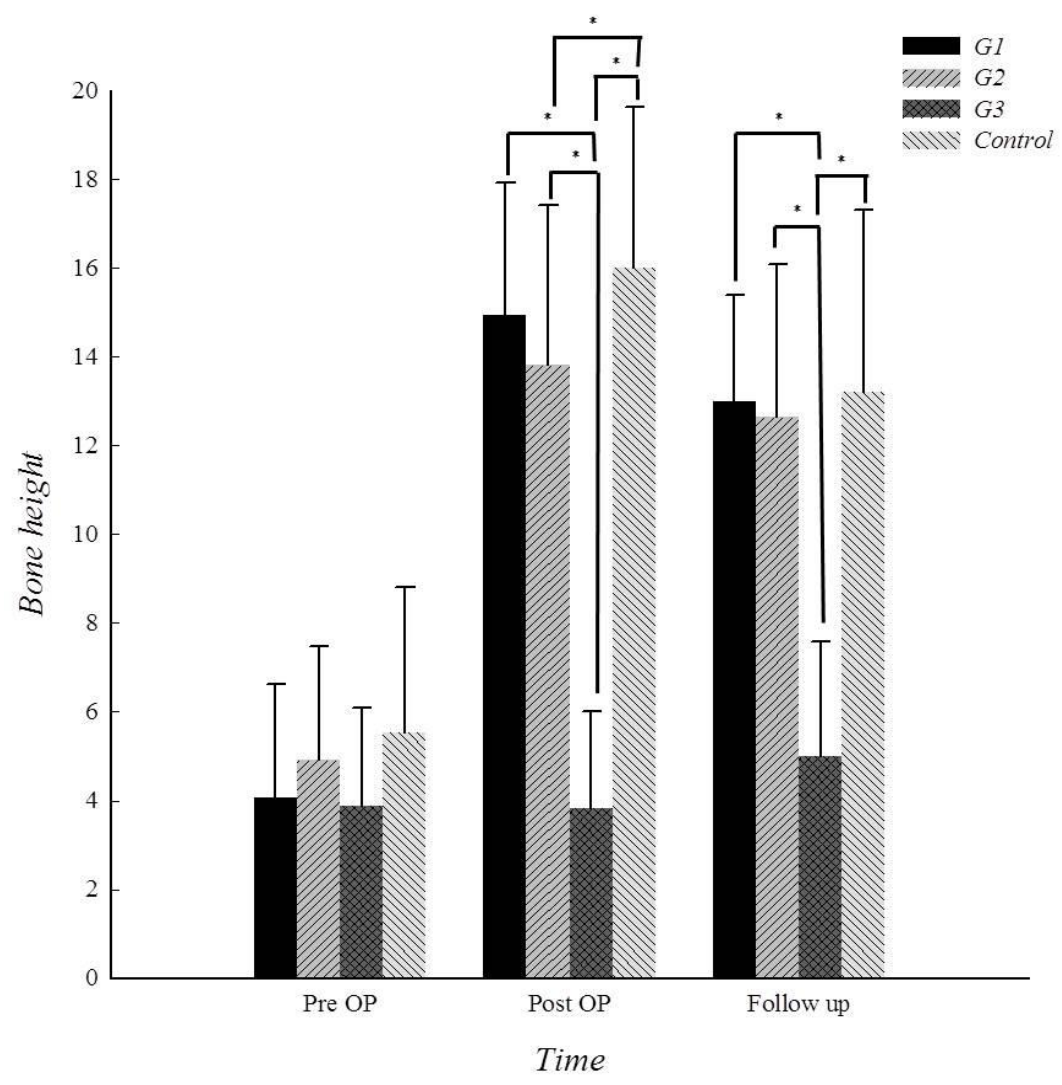

Figure 5. Graph of Tables 3 and 4. * indicates a significant difference in comparison with variable values at $p<0.016$. Significance was determined using two-way repeated-measures ANOVA. Significance for pairwise comparison was determined with the Bonferroni post hoc test. Data are presented as means \pm standard deviations.

\section{Discussion}

SMP is the most common complication to occur during sinus floor elevation procedures and must be managed to prevent loss of valuable bone graft and bone substitute materials [9]. Various treatments have been proposed for the repair of SMPs.

CMs are widely used in the treatment of SMPs. Although fast-resorbing CMs are often used, they possess no rigidity and, as a result, may be not adequate for large membrane perforations [10, 11]. Nevertheless, small membrane repairs using fast-resorbing CMs have been well documented. A fast-resorbing CM is a soft, white, pliable, and nonfriable sponge that is often used in dental surgery. It can be the better choice for the repair of small-to-medium-sized SMPs $(<10 \mathrm{~mm})$ for controlling bleeding, stabilizing blood clots, as well as protecting the membrane while accelerating the healing process more predictably than slow-resorbing CMs [12]. As this current study shows, when there is an SMP, the bone graft could still be completed following the CM repair, and the implants could be placed in most of the patients without complications. There were no significant differences in acquired bone heights compared with patients without perforations at the various follow-up time points. Those differences that exist in bone heights at the postoperative time points can be assumed to be due to the fact that further mucosal elevation is more carefully performed when a perforation occurs during the sinus lifting procedure.

Large perforations $(>10 \mathrm{~mm})$ are the most difficult and challenging to repair during sinus lift surgery. Suturing large SMPs can be difficult because of the limited access and the friability of the maxillary sinus membrane. Also, the membrane edges of the torn mucosa cannot be approximated in the case of large SMPs [11,13]. Here, a slow-resorbing CM can be placed on the SMP in order to make a pouch [14]. This pouch is meant to surround and isolate the bone graft. However, such isolation 
using a pouch might have an adverse effect on the neovascularization of the torn mucosa healing and graft incorporation. When the membrane perforation is large or cannot be controlled, stopping the procedure and reentering after 6-8 weeks to allow for the regeneration of the sinus membrane is one option [15]. The authors of this study have inserted CPs into cases with large maxillary sinus tears without additional bone graft in cases with large SMPs. There are other studies which have proposed that an isolated space created by the elevated membrane and a simultaneous protruding implant placement without insertion of any bone substitute resulted in new bone formation [16-19]. A CP can therefore act as additional space-making material between the sinus membrane and sinus floor. In the present study, the authors could observe bone gain after CP repair in cases of large SMPs. However, this was only about $1 \mathrm{~mm}$ compared with the preoperative time point. This may be insufficient to allow implant placement with full bone coverage-in other words, impossible without a bone graft.

Pathological conditions, which include the presence of sinusitis or inflammatory-induced membrane changes, might be potential risk factors for SMPs during sinus lift procedures [5]. Sometimes the authors have found that these unhealthy membranes are not perforated during the sinus lift procedure but they are weakened, thinned, or irritated. Such weakened membranes can potentially develop later perforations that can lead to bone graft failure after procedure. In order to avoid this, the authors have used CMs for reinforcement of weakened sinus membranes. The use of CMs may prove to be useful for protecting such potentially weakened sinus membranes.

The results of the present study suggest, therefore, that CMs are appropriate materials for small-to-moderate-sized SMPs or to manage weakened sinus membranes. Inserting only a CP to manage large SMPs can be insufficient without additional bone gaining for dental implant placement. However, further studies are required to confirm the biological interaction of the bone around the dental implant. These studies would involve a large sample size, randomization, and sufficient long-term follow-up.

Author Contributions: J.-Y.L. carried out the analysis of data and prepared the manuscript. J.-J.K. helped in the collection and analysis of the data. G.K.S. conceived of the study, participated in its design and coordination, and helped to draft the manuscript. Y.-D.K. designed the study and drafted the manuscript.

Funding: This study was supported by the National Research Foundation of Korea (NRF) grant funded by the Korea government (MSIT) (NRF-2018R1A5A2023879) and a grant of the Korea Health Technology R\&D Project through the Korea Health Industry Development Institute (KHIDI), funded by the Ministry of Health \& Welfare, Republic of Korea (HI17C0708).

Conflicts of Interest: The authors declare no conflict of interest.

\section{References}

1. Tatum, H., Jr. Maxillary and sinus implant reconstructions. Dent. Clin. North. Am. 1986, 30, $207-229$. [PubMed]

2. Boyne, P.J.; James, R.A. Grafting of the maxillary sinus floor with autogenous marrow and bone. J. Oral. Surg. 1980, 38, 613-616. [PubMed]

3. Stern, A.; Green, J. Sinus lift procedures: An overview of current techniques. Dent. Clin. North. Am. 2012, 56, 219-233. [CrossRef] [PubMed]

4. Katranji, A.; Fotek, P.; Wang, H.L. Sinus augmentation complications: Etiology and treatment. Implant. Dent. 2008, 17, 339-346. [CrossRef] [PubMed]

5. Al-Dajani, M. Incidence, Risk Factors, and Complications of Schneiderian Membrane Perforation in Sinus Lift Surgery: A Meta-Analysis. Implant. Dent. 2016, 25, 409-415. [CrossRef] [PubMed]

6. Fugazzotto, P.A.; Vlassis, J. A simplified classification and repair system for sinus membrane perforations. J. Periodontol. 2003, 74, 1534-1541. [CrossRef] [PubMed]

7. Choi, B.H.; Zhu, S.J.; Jung, J.H.; Lee, S.H.; Huh, J.Y. The use of autologous fibrin glue for closing sinus membrane perforations during sinus lifts. Oral. Surg. Oral. Med. Oral. Pathol. Oral. Radiol. Endod. 2006, 101, 150-154. [CrossRef] [PubMed]

8. An, J.H.; Park, S.H.; Han, J.J.; Jung, S.; Kook, M.S.; Park, H.J.; Ryu, S.Y.; Oh, H.K. Treatment of dental implant displacement into the maxillary sinus. Maxillofac. Plast. Reconstr. Surg. 2017, 39, 35. [CrossRef] [PubMed] 
9. Pikos, M.A. Maxillary sinus membrane repair: Report of a technique for large perforations. Implant. Dent. 1990, 8, 29-34. [CrossRef]

10. Proussaefs, P.; Lozada, J.; Kim, J.; Rohrer, M.D. Repair of the perforated sinus membrane with a resorbable collagen membrane: A human study. Int. J. Oral. Maxillofac. Implants. 2004, 19, 413-420. [PubMed]

11. Pikos, M.A. Maxillary sinus membrane repair: Update on technique for large and complete perforations. Implant. Dent. 2008, 17, 24-31. [CrossRef] [PubMed]

12. Yu, S.J.; Na, I.C.; Jang, H.W.; Jung, M.J.; Kim, B.O. The increase of keratinized and attached gingiva using collagen wound dressing in dogs: A clinical and histomorphometric comparative study. Oral. Biology Research. 2015, 39, 10-17.

13. de Oliveira, H.A.A.B.; de Moraes, R.P.F.; Limirio, P.H.J.O.; Dechichi, P. Repair of a perforated sinus membrane with an autogenous periosteal graft: A study in 24 patients. Br. J. Oral. Maxillofac. Surg. 2018, 56, 299-303. [CrossRef] [PubMed]

14. Proussaefs, P.; Lozada, J. The "Loma Linda pouch": A technique for repairing the perforated sinus membrane. Int. J. Periodontics Restorative Dent. 2003, 23, 593-597. [PubMed]

15. Chanavaz, M. Maxillary sinus: Anatomy, physiology, surgery and bone grafting related to implantology—eleven years of surgical experience (1979-1990). J. Oral. Implantol. 1990, 16, 199-209. [PubMed]

16. Cricchio, G.; Palma, V.C.; Faria, P.E.P.; de Olivera, J.A.; Lundgren, S.; Sennerby, L.; Salata, L.A. Histological outcomes on the development of new space-making devices for maxillary sinus floor augmentation. Clin. Implant. Dent. Relat. Res. 2011, 13, 224-230. [CrossRef] [PubMed]

17. Lundgren, S.; Andersson, S.; Gualini, F.; Sennerby, L. Bone reformation with sinus membrane elevation: A new surgical technique for maxillary sinus floor augmentation. Clin. Implant. Dent. Relat. Res. 2004, 6, 165-173. [CrossRef] [PubMed]

18. Thor, A.; Sennerby, L.; Hirsch, J.M.; Rasmusson, L. Bone formation at the maxillary sinus floor following simultaneous elevation of the mucosal lining and implant installation without graft material: An evaluation of 20 patients treated with 44 Astra Tech implants. J. Oral. Maxillofac. Surg. 2007, 65, 64-72. [CrossRef] [PubMed]

19. Sul, S.H.; Choi, B.H.; Li, J.; Jeong, S.M.; Xuan, F. Effects of sinus membrane elevation on bone formation around implants placed in the maxillary sinus cavity: An experimental study. Oral. Surg. Oral. Med. Oral. Pathol. Oral. Radiol. Endod. 2008, 105, 684-687. [CrossRef] [PubMed] 